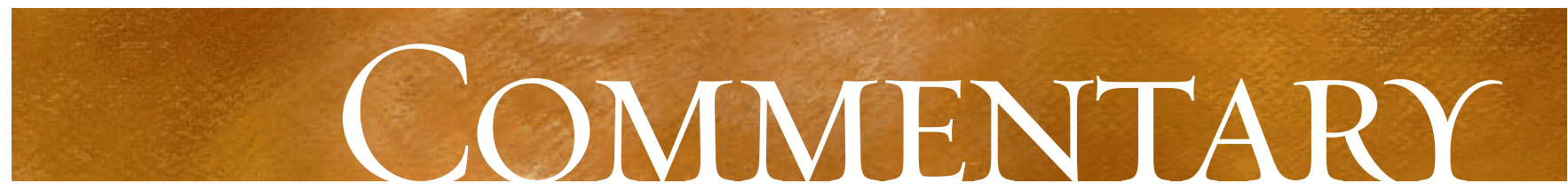

\title{
Low risk of depression in diabetes? Would that it were so
}

\author{
Deborah J. Wexler
}

$\infty \quad$ See related article page 42

$\mathrm{P}$ ractitioners who care for patients with diabetes are familiar with the problems of comorbid depression: decreased compliance with treatment, increased risk of complications and disability, and diminished health-related quality of life. The large body of data outlined by Brown and colleagues $^{1}$ has demonstrated that the prevalence of depression is increased in diabetes and vice-versa. How, then, are we to understand their finding ${ }^{1}$ that type 2 diabetes does not increase the risk of depression?

Using administrative databases that capture $99 \%$ of Saskatchewan's population, these authors identified 3I 635 adults with type 2 diabetes newly diagnosed between 1992 and 2000 , and followed them for an average of 4.5 years. Patients with type 2 diabetes were identified by outpatient or inpatient service codes for diabetes; gestational diabetes was excluded. The study cohort was limited to those dispensed a hypoglycemic drug to be taken orally; subjects who met the case definition within the preceding 3 years ( 1989 through I99I) were excluded. Controls were 57 I4I people without diabetes who were randomly selected from the database and matched to cases only by the date of identification of diabetes.

Similarly, patients in the cohort who experienced depression were identified by the diagnosis codes for depressive disorders and prescriptions for antidepressant medications. Again, patients who met this definition during the 3 years preceding the study period were excluded. With this method, a greater proportion of cases ( $4.9 \%$ of patients with diabetes) than controls $(3.8 \%$ of patients without diabetes) were excluded from the study cohort because of prior depression. In the remaining cohort, the authors modelled the hazard ratio of new-onset depression according to presence of diabetes both in crude models and in models adjusted for age, sex, number of physician visits, insulin usage and comorbidities (including arthritis, cancer, stroke, coronary artery disease and peripheral arterial disease).

With these selection criteria, the authors included subjects with generally recent-onset, mild type 2 diabetes, and excluded depression-prone subjects (i.e., those with a history of depression). Although type 2 diabetes can be quite advanced $\approx$ at the time of diagnosis, most patients with type 2 diabetes do not develop serious complications within 5 years of diagnosis. Moreover, by including only subjects treated with oral hypoglycemics, Brown's group excluded as well those patients with severe comorbidities (e.g., renal failure, heart failure) that preclude oral hypoglycemic use; such comorbidities are known to be associated with depression. As a result of their

selection criteria, the authors have suggested that patients with relatively recent-onset diabetes, and with little in the way of advanced complications, are not at higher risk for depression than people without diabetes.

However, their data belie their title, since even patients with new-onset diabetes had an increased risk of depression; those with more severe diabetes had an even higher risk. In this study's Saskatchewan population, patients with diabetes had a statistically significant 10\% increased risk of developing depression in crude analyses. Patients treated with insulin - which the authors appropriately consider to be a proxy for severity of disease - had an unadjusted $34 \%$ increase in their risk of developing depression. After adjusting for peripheral arterial disease (and thereby controlling for the effect of severe complications), diabetes alone was no longer associated with increased risk of depression, whereas complications of diabetes marked by the presence of peripheral arterial disease were.

\section{Brown's group excluded subjects prone to depres- sion as well as patients with severe comorbidities.}

Other studies have shown that mild, asymptomatic, type 2 diabetes is not associated with depression or impaired quality of life, whereas symptomatic comorbidities and insulin use appear to be risk factors. ${ }^{2-5}$ Population-based studies, as opposed to those carried out in clinical settings, have shown relatively high quality of life in patients with type 2 diabetes. ${ }^{6,7}$ Brown and colleagues' results are consistent with those of prior studies and suggest that although asymptomatic early diabetes may not increase the risk for depression, the risk of depression is increased when all patients with diabetes are taken into account.

The onset of type 2 diabetes is far more insidious than many other diseases such as stroke, which has an abrupt and devastating onset, or arthritis, which is usually diagnosed after pain has developed; both of these conditions are strongly associated with depression in this and other studies. In the absence of hyper- or hypoglycemia, patients with early type 2 
diabetes may be asymptomatic. Once complications emerge, the prevalence and risk of depression climbs. Brown and colleagues' findings remind us that we should focus our attention among patients newly diagnosed with diabetes on prevention of complications, while at the same time remaining sensitive to the risk of depression if, despite all efforts, complications of diabetes develop.

This article has been peer reviewed.

Deborah J. Wexler is with the Department of Endocrinology, Massachusetts General Hospital, Boston, Mass.

Competing interests: None declared.

\section{REFERENCES}

I. Brown LC, Majumdar SR, Newman SC, et al. Type 2 diabetes does not increase risk of depression. CMAJ 2006;175(I):42-6.
2. Wexler DJ, Grant RW, Wittenberg E, et al. Correlates of health-related quality of life in type 2 diabetes. Diabetologia DOI: I0.1007/soor25-006-0249-9. Epub 2006 Apr 29 ahead of print.

3. UK Prospective Diabetes Study Group. Quality of life in type 2 diabetic patients is affected by complications but not by intensive policies to improve blood glucose or blood pressure control (UKPDS 37). Diabetes Care 1999;22:1125-36.

4. Redekop WK, Koopmanschap MA, Stolk RP, et al. Health-related quality of life and treatment satisfaction in Dutch patients with type 2 diabetes. Diabetes Care 2002;25:458-63

5. Bell RA, Smith SL, Arcury TA, et al. Prevalence and correlates of depressive symptoms among rural older African Americans, Native Americans, and whites with diabetes. Diabetes Care 2005;28:823-9.

6. Manuel DG, Schultz SE. Health-related quality of life and health-adjusted life expectancy of people with diabetes in Ontario, Canada, 1996-1997. Diabetes Care 2004;27:407-I4

7. Maddigan SL, Feeny DH, Johnson JA. Health-related quality of life deficits associated with diabetes and comorbidities in a Canadian National Population Health Survey. Qual Life Res 2005;I4:131I-20.

Correspondence to: Dr. Deborah Wexler, Department of Endocrinology, Massachusetts General Hospital, 55 Fruit St., Boston MA 02II4, USA; dwexler@partners.org

\title{
Clinical practice guidelines for the use of parathyroid hormone in the treatment of osteoporosis
}

\author{
Anthony Hodsman for the Scientific Advisory Council of Osteoporosis Canada, Alexandra \\ Papaioannou for the Clinical Guidelines Committee, Ann Cranney for the Writing Group \\ on the Systematic Review of Parathyroid Hormone for the Treatment of Osteoporosis
}

See related article page 52

I n 2002, Osteoporosis Canada published clinical practice guidelines for the diagnosis and management of osteoporosis in Canada. ${ }^{1}$ At the time, there was considerable evidence for the use of anti-resorptive agents in the management of osteoporosis, including estrogen, the selective estrogen receptive modulator, raloxifene, several approved bisphosphonates (alendronate, etidronate and risedronate) and calcitonin. For these agents, much information exists to document their primary efficacy in preventing fractures, the most important outcome of osteoporosis.

In contrast to anti-resorptive drugs, anabolic agents result in the formation of new bone in both trabecular and cortical envelopes and thus partially repair the deterioration in micro architecture that leads to the increased fragility of adult osteoporotic bone. Sodium fluoride was the first of such agents to be evaluated in randomized controlled trials, but the results showed that the bone of patients receiving the drug was of poor quality and that the incidence of fractures was not reduced even though large measurable increments in bone mineral density (BMD) were seen. In the 2002 guidelines, parathyroid hormone (PTH) was mentioned only briefly because the pivotal phase III trial of teriparatide (PTH[ I-34]) had only just been published, ${ }^{2}$ and regulatory approval for its clinical use had not yet been obtained in Canada.

Osteoporosis Canada updates its clinical guidelines at intervals, when a sufficient body of evidence becomes available to allow a considered interpretation of the place new therapies should occupy in the management of osteoporosis. It has now been possible for the Clinical Guidelines Committee of Osteoporosis Canada to perform a systemic review of trials evaluating PTH for the treatment of osteoporosis. Its findings appear in this issue of $C M A J$ (see page 52). ${ }^{3}$ Both the systematic review and the recommended guidelines were reviewed by the Scientific Advisory Council of Osteoporosis Canada (members of which include primary care physicians), together with a panel of patients with osteoporosis. Teriparatide has been most widely used. There is only limited information on the full sequence hormone PTH(I-84), given that the results of the phase III antifracture trial have yet to be published in full. Another analogue, cyclic $\mathrm{PTH}(\mathrm{I}-3 \mathrm{I})$ amide, is currently undergoing phase II evaluation. 